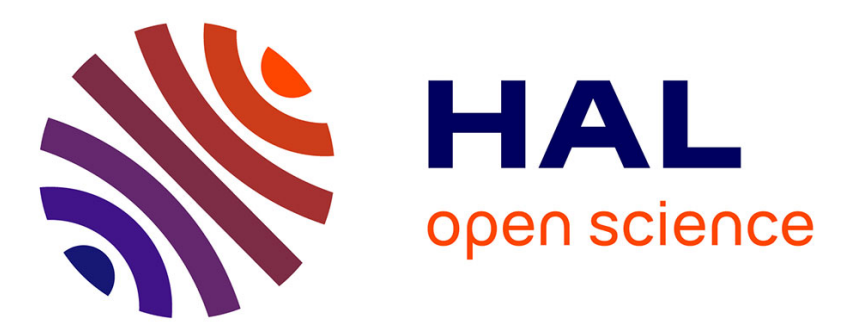

\title{
Probing the Growth of Organic Molecular Films Embedded between Cobalt and Iron Electrodes: Ferromagnetic Nuclear Resonance Approach
}

Garen Avedesian, Jacek Arabski, Jennifer Wytko, Jean Weiss, Christian Meny

\section{- To cite this version:}

Garen Avedesian, Jacek Arabski, Jennifer Wytko, Jean Weiss, Christian Meny. Probing the Growth of Organic Molecular Films Embedded between Cobalt and Iron Electrodes: Ferromagnetic Nuclear Resonance Approach. Advanced Functional Materials, 2020. hal-02927737

\section{HAL Id: hal-02927737 \\ https://hal.science/hal-02927737}

Submitted on 1 Sep 2020

HAL is a multi-disciplinary open access archive for the deposit and dissemination of scientific research documents, whether they are published or not. The documents may come from teaching and research institutions in France or abroad, or from public or private research centers.
L'archive ouverte pluridisciplinaire HAL, est destinée au dépôt et à la diffusion de documents scientifiques de niveau recherche, publiés ou non, émanant des établissements d'enseignement et de recherche français ou étrangers, des laboratoires publics ou privés. 
Probing the growth of organic molecular films embedded between cobalt and iron electrodes: Ferromagnetic nuclear resonance approach

Garen Avedissian*, Jacek Arabski, Jennifer A. Wytko, Jean Weiss and Christian Meny*

G. Avedissian, J. Arabski, Dr. C. Meny

Institut de Physique et Chimie des Matériaux de Strasbourg, Université de Strasbourg, CNRS UMR 7504, 67200 Strasbourg, France.

E-mail : christian.meny@ipcms.unistra.fr

E-mail : garen.avedissian@ipcms.unistra.fr

Prof. J. Weiss, Dr. J. A. Wytko

Institut de Chimie de Strasbourg, Université de Strasbourg, CNRS UMR 7177, 67000 Strasbourg, France.

Keywords: Organics, nanostructures, solid-state NMR, growth, ferromagnetism

\section{Abstract}

Physical properties of magnetic nanostructures and devices strongly depend on the morphological characteristics of their various components. This is especially true and becomes particularly complex in hybrid nanostructures, where soft organic molecules are at the vicinity of ferromagnetic metallic films. The supramolecular architecture of molecular films embedded between iron $(\mathrm{Fe})$ and cobalt $(\mathrm{Co})$ layers, has been investigated by ferromagnetic nuclear resonance (FNR). In such sample architecture, the presence of pin holes in the organic layers is detected by FNR contributions in a specific spectral range. The methodology that has been developed allows probing the continuity and packing of zinc tetra-phenyl porphyrin (ZnTPP) molecular films between the Co and Fe films. The experimental results suggest that, regardless of the nature of the ferromagnetic under-layer, at least 15 monolayers of ZnTPP are necessary to form continuous and pin-hole free molecular films. In addition, quantitative analyses show that ZnTPP layers exhibit distinct morphologies that are dependent on the nature of the ferromagnetic metallic under-layer.

\section{Introduction}


Interface induced magnetic effects are a main pillar for modern technology. They have already been widely studied in metal and metal oxide-based devices and nowadays extensive work is performed on hybrid architectures where organic molecules are combined with metallic and/or metal oxide layers. One of the main difficulties in studying metal/organic heterostructures is the control of the organic layers that originates from the soft, light-weight character of the organic molecules. The complex behaviour of organic molecules on metallic surfaces can also lead to artefacts like exchange bias attributed to the molecular films ${ }^{[1,2]}$ that was also observed in the samples under investigation here, but revealed to be due to the partial oxidation of the cobalt film. Similarly, devices based on organic tunnel junctions revealed, surprisingly, tunnel magnetoresistance (MR) effect for barrier thickness that is an order of magnitude thicker than the distance where one would expect to see tunnelling effects. ${ }^{[3]}$ A key factor essential to reliable experiments is to first ascertain that the molecular layers form continuous films on top of the metallic electrode and once built, prevent the migration of metallic atoms between the electrodes embedding the organic films. The growth of organic films on top of metallic films has been extensively studied especially by near field methods. ${ }^{[4]}$ However, these techniques are more difficult to implement when a second metallic layer is deposited on top of the organic film. To our knowledge, once organic films are embedded between ferromagnetic films, few investigation methods are available which allow to probe the already grown molecular films. In addition, the mechanical softness of the organic films complicates the implementation of some experimental techniques like transmission electron microscopy (TEM). Indeed, such techniques require post-growth sample thinning preparation that might easily degrade the molecular films. To achieve a deeper understanding of the morphology of embedded organic films, ferromagnetic nuclear resonance (FNR) ${ }^{[5]}$ experiments have been performed and an original analyses methodology has been developed. This approach allows investigating the morphology of organic layers embedded between ferromagnetic Co and Fe electrodes. Co and Fe films are very common ferromagnetic electrodes for spintronic devices. Therefore, the 
$\mathrm{Co} /$ organic/Fe stacks studied in this work can be considered as basic building blocks for such kind of devices.

FNR corresponds to nuclear magnetic resonance (NMR) applied to ferromagnetic systems and is also sometime called zero field NMR or internal field NMR. Although NMR is widely used in biology or chemistry, its use for studying ferromagnetic nanostructures is much scarcer. However, it has been shown that FNR is a very efficient tool to study the structure and interface morphology of thin films ${ }^{[6,7,8,9]}$ multilayers ${ }^{[10.11,12,13,14]}$ or even devices. ${ }^{[15]}$ FNR also provides unique insight into the understanding of physicochemical properties of assemblies of nanoobjects. ${ }^{[16,17,18,19,20]}$ In this work, an original FNR methodology is applied to study organic films, namely, molecular layers of zinc tetra-phenyl porphyrin (ZnTPP). Compared to the widely studied phthalocyanines, tetra-phenyl porphyrins are non-planar molecules. Therefore, compared to phthalocyanines, tetraphenyl-porphyrins are expected to have different behaviour when adsorbed on metallic surfaces due to the occurrence of different molecule/surface interactions. The FNR method that has been developed showed that a minimum of 15 molecular layers are required to obtain continuous organic films in $\mathrm{Co} / \mathrm{ZnTPP} / \mathrm{Fe}$ and $\mathrm{Fe} / \mathrm{ZnTPP} / \mathrm{Co}$ heterostructures. In addition, the quantitative analyses of the FNR spectra allowed to determine the evolution of the morphology of the ZnTPP molecular layers with the organic layer thickness. Even though the threshold thickness of ZnTPP required to obtain continuous films when it is deposited on top of Co (15ML) is very close to the one obtained when it is deposited on top of Fe film (14ML); on Co the ZnTPP layers initially show a layered morphology, whereas on Fe, ZnTPP molecules form isolated islands. The experimental data also suggest that the migration of metallic atoms through the molecular film takes place. This work demonstrates that the described FNR methodology is very efficient to investigate the continuity of organic films embedded between Co and Fe layers and can be applied to any type of spacer layer films.

\section{Results and discussion}


The FNR methodology that has been developed is based on the sensitivity of the Co FNR resonance frequency to its nearest neighbour $(\mathrm{NN})$ cell chemical environment. Indeed, when non-ferromagnetic elements are included in the Co NN cell, the Co resonance frequency is lowered compared to its resonance frequency when surrounded by other Co atoms only. ${ }^{[21,22]}$ In contrast, when a Co atom nears Fe atoms in its NN cell, its resonance frequency is enhanced. ${ }^{[21,22]}$ Therefore, as shown in Figure 1, the FNR spectrum of a $\mathrm{Cu} / \mathrm{Co} / \mathrm{Fe}$ stack can be divided into three frequency ranges: one for the bulk part of the probed Co atoms (Co atoms surrounded by $\mathrm{Co}$ atoms only: between 200 and $240 \mathrm{MHz})$, one for the $\mathrm{Cu} / \mathrm{Co}$ interfacial region $(<200$ $\mathrm{MHz}$ ) and one for the $\mathrm{Co} / \mathrm{Fe}$ interfacial region $(>240 \mathrm{MHz}$ ). Therefore, when inserting an organic molecular layer between the Co and Fe films, it is straightforward to probe whether the molecular layer is continuous and/or if $\mathrm{Fe}$ or Co atoms migrate through the organic layer. Indeed, once the organic layer completely isolates the two ferromagnetic electrodes, the high frequency (>240 MHz) contribution in the FNR spectrum will vanish.

This effect is shown in Figure 2 where the FNR high frequency spectral range of $\mathrm{Cu}(25 \mathrm{~nm}) /$ Co $(6 \mathrm{~nm}) / \mathrm{ZnTPP}(t$ in $M L) / \mathrm{Fe}(30 \mathrm{~nm})$ samples; $1 \mathrm{ML}<\mathrm{ZnTPP}<60 \mathrm{ML}$, are compared with the $\mathrm{Cu}(25 \mathrm{~nm}) / \mathrm{Co}(6 \mathrm{~nm}) / \mathrm{Fe}(30 \mathrm{~nm})$ reference sample (i.e. no ZnTPP layer between Fe and Co films, means full diffusion of the Fe to the Co electrode). The full frequency range spectra are given for information in Figure S1. As expected, the Co-Fe contribution decreases when the ZnTPP thickness increases and eventually vanishes. A similar behaviour has been observed for samples with $3 \mathrm{~nm}$ of cobalt film (Figure S2). Using the same methodology, the behaviour of ZnTPP on top of Fe has been investigated in heterostructures that have the following stack: $\mathrm{Cu}(25 \mathrm{~nm}) / \mathrm{Fe}(30 \mathrm{~nm}) / \mathrm{ZnTPP}(t$ in $M L) / \mathrm{Co}(6 \mathrm{~nm})$; with ZnTPP thicknesses of 3, 6, 12 and 15 ML. The vanishing intensity at high frequency is also observed in these samples (Figure S3) which confirms the validity of the interpretation explained above. 
To obtain more quantitative analyses, the coverage ratio of the ZnTPP layers was estimated from the integral intensities of the high frequency area of the FNR spectra.

$$
C=\left(1-\frac{I n t_{C o / Z n T P P / F e}}{I n t_{C o / F e}}\right) \times 100
$$

Where

- $\quad C$ is the coverage ratio in percentile $(\%)$.

- $\quad$ Int $t_{C o / Z n T P P / F e}$ is the integral intensity of the spectra (above $240 \mathrm{MHz}$ ) for the $\mathrm{Cu} / \mathrm{Co}$ $(6 \mathrm{~nm}) / \mathrm{ZnTPP}(t$ in $M L) / \mathrm{Fe}(30 \mathrm{~nm})$ and $\mathrm{Cu} / \mathrm{Co}(3 \mathrm{~nm}) / \mathrm{ZnTPP}(t$ in $M L) / \mathrm{Fe}(30 \mathrm{~nm})$ samples.

- Int $_{\mathrm{Co} / \mathrm{Fe}}$ is the integral intensity of the spectrum (above $240 \mathrm{MHz}$ ) for the $\mathrm{Cu} / \mathrm{Co} / \mathrm{Fe}$ reference sample.

The evolution of ZnTPP coverage ratio with the increase of the molecular thickness is shown in Figure 3. When ZnTPP is deposited on top of Co (black symbol in Figure 3) the coverage ratio increases rapidly with the first adsorbed monolayer of ZnTPP and then shows a slow asymptotic increase that reaches full coverage at almost the $15^{\text {th }} \mathrm{ML}$ of the molecule. On the contrary, when ZnTPP is deposited on top of Fe (red symbol in Figure 3) the molecular coverage increases linearly with the ZnTPP thickness and exhibits 14 ML of ZnTPP as threshold thickness for full coverage. These analyses show that the morphology of ZnTPP molecular layers strongly depend on the nature of the underneath metallic layer. The thickness to obtain complete separation i.e. pin hole free, of the two electrodes is quite large for both underlayers and is in the range of $14-15 \mathrm{ML}$.

To better understand the evolution of the coverage ratio with the increase in ZnTPP thickness, one should consider the stacking of the ZnTPP films on top of metallic layers. Figure 4 shows a schematic view of one, two and three monolayers of organized ZnTPP adsorbed on top of a 
metallic surface. For the proposed model, several points are considered: (i) a square metal surface lattice has been chosen for simplicity, (ii) it is assumed that the 4 phenyl rings are almost perpendicular to the porphyrin plane i.e. perpendicular to the surface, (iii) the arrangement of the molecules experience small deviation from the crystal structure of ZnTPP due to the surface/molecule interactions (weak van der Waals forces) (iv) the molecular ordering is arranged as it respects the need for maximum $\pi-\pi$ stacking between the ZnTPP molecules. As observed from Figure 4a, a complete monolayer of ZnTPP would leave large amounts of uncovered metallic atoms. From such a scheme, by simply considering the area of uncovered metallic surface, it is possible to estimate that a complete single layer of ZnTPP would cover only about $62 \%$ of the metallic surface atoms. Therefore, full separation of the top and bottom electrodes can only be achieved by the stacking of additional ZnTPP layers. The $62 \%$ coverage deduced from the scheme in Figure $4 \mathrm{a}$ is close to that obtained in the analysis of the coverage ratio in Figure 3 for the smallest thickness (i.e. 1 ML) of ZnTPP deposited on top of Co. This suggests that the ZnTPP molecules on top of Co, at least for the first few organic layers, have a layered morphology. On the contrary, when ZnTPP molecules are deposited on top of Fe, the coverage ratio increases linearly, at a small rate of $7 \%$ for additional coverage per ML. This observation is not compatible with a layered morphology and suggests that when ZnTPP is deposited on top of Fe, it forms islands.

On Co, once the first ZnTPP layer is completed, the number of voids remaining after the deposition of the subsequent layers depends on how these layers stack on top of the first one. The stacking depicted in Figure $\mathbf{4 b}$ and $\mathbf{4 c}$ represent the additional second and third monolayers, respectively. The subsequent monolayers are modelled so that the ZnTPP molecules respect the offset typically observed in J-aggregate kind of arrangement. ${ }^{[23,24]}$ In consequence, if ZnTPP layers follow this kind of stacking, the coverage ratio would increase to about $85 \%$ with the second monolayer and to almost $100 \%$ with the third one. These computed 
values are larger than that determined experimentally in Figure 3 and implies that already for the third layer of ZnTPP, bottom and top electrodes would be fully separated. This is obviously not the case experimentally because, as discussed for the analysis of Figure 3, 14-15 ML of ZnTPP are required to separate the electrodes. These observations and analyses suggest that, on a Co film, the morphology of the ZnTPP films does not show a purely layered structure when the thickness is increased above 1ML.

Considering the complexity of the layering process, we have chosen to describe the coverage ratio of ZnTPP on top of Co in a phenomenological way, considering that each subsequent ZnTPP layer decreases the number of remaining voids between the Co and Fe layers by a constant ratio using the formula:

$C_{\text {Phenom }}(n)=\left(1-0.38 \times(1-f)^{n-1}\right) \times 100 ; n>1$

where:

- $C_{\text {Phenom }}(n)$ is the coverage ratio in $(\%)$ as determined by the phenomenological model.

- 0.38 is the fraction of voids remaining on top of the metallic surface after completion of the first ZnTPP layer.

- $\quad f$ is the fraction of voids that are filled in each subsequent layer.

- $\quad n$ is the number of deposited layers.

The closest simulation is shown in Figure 3 by the full black line and is obtained by considering that each subsequent layer fills only $20 \%$ of the remaining voids $(f=0.2)$. In this model, the full separation of the two electrodes is only reached asymptotically. For a deposited ZnTPP thickness of $15 \mathrm{ML}$, the coverage ratio would reach $98 \%$ of the surface of the underlayer. This fraction of voids filling per layer " $f$ ' is surprisingly low and can hardly be explained only by the onset of an island morphology after the completion of the first ZnTPP layer. Therefore, it is 
most likely that migration of $\mathrm{Co}$ and $\mathrm{Fe}$ atoms through the organic layers does also take place. It is most probably also the case when ZnTPP is deposited on top of the Fe film.

To investigate the influence of the continuity of the organic layers on the physical properties of the heterostructures, the magnetization curves of the same stacks were measured. Figure 5 shows the magnetization curves of the $\mathrm{Cu} / \mathrm{Co} / \mathrm{Fe}$ reference sample, of a sample well below the threshold thickness $(\mathrm{Cu} / \mathrm{Co} / \mathrm{ZnTPP}(3 \mathrm{ML}) / \mathrm{Fe})$ and of a sample well above the threshold $(\mathrm{Cu} / \mathrm{Co} / \mathrm{ZnTPP}(24 \mathrm{ML}) / \mathrm{Fe})$. No strong difference can be detected in the magnetization curves of the three samples. This lack of difference shows that it is not possible to identify the discontinuity of organic layers from such magnetic measurements and confirms the effectiveness of our approach. Comparison of the saturation magnetization values showed that all the samples are well below the expected value, considering the thicknesses of the deposited $\mathrm{Co}(6 \mathrm{~nm})$ and $\mathrm{Fe}(30 \mathrm{~nm})$ layers. Indeed, for these thicknesses, saturation magnetization should be $0.006 \mathrm{emu} . \mathrm{cm}^{-2}$, and even for the $\mathrm{Cu} / \mathrm{Co} / \mathrm{Fe}$ reference sample, it is only of the order of 0.0045 emu. $\mathrm{cm}^{-2}$. This difference is attributed to the dead layers at the $\mathrm{Fe} / \mathrm{Cr}$ interface. In addition, when ZnTPP layers are introduced between the Co and Fe layers, the saturation magnetization decreases further. Although this decrease is close to the experimental measurement error bars, it is probably related to the migration of the Co and Fe atoms through the organic layers as suggested above. These isolated atoms or small clusters do not contribute to the saturation magnetization. A closer look at the shape of the magnetization curves reveals that the $\mathrm{Cu} / \mathrm{Co} / \mathrm{Fe}$ reference sample shows a low coercive field due to the large thickness of Fe and a larger saturation field due to the magnetically stiffer Co layer. Samples with ZnTPP thicknesses below (3ML) and above (24 ML) the threshold value display similar magnetic behaviour, with magnetization curves having a two-step reversal process. Considering the magnitude of the magnetization reversal, the low field reversal can be attributed to the Fe $(30 \mathrm{~nm})$ layer whereas the second reversal at higher fields corresponds to the thinner and magnetically stiffer Co (6 
$\mathrm{nm}$ ) layer. The $3 \mathrm{ML}$ sample shows a coercive field similar to the one of the reference sample what is consistent with the fact that the two magnetic layers are directly coupled through pinholes in the organic film. The $24 \mathrm{ML}$ sample shows a reversal field for Fe that is larger than those of the previous two samples (Figure 5 black curve (reference) and blue curve (3ML)). This difference could be due to a greater stiffness of the top Fe layer due to increase in the interface $(\mathrm{ZnTPP} / \mathrm{Fe})$ roughness induced by the increase in ZnTPP thickness. The increasing roughness might result in additional magnetic pinning centres resulting in larger reversal fields. The increased coercive field could also originate from an indirect exchange coupling between the Co and Fe layers through the organic film. The coercive field of the soft Fe layer would be increased by a ferromagnetic coupling with the stiffer Co film. It will be investigated in a future work using samples with better balanced Fe and Co thicknesses. Finally, even if the magnetic measurements were performed at $2 \mathrm{~K}$ after a field cooling procedure, all the magnetization curves were perfectly symmetric. No exchange bias, as in other metal/organic systems ${ }^{1,2}$, was observed in these samples at least when they were measured freshly after deposition. However, only after some time of exposure to air, the onset of exchange bias appears. The onset of exchange bias is attributed to the aging of the samples that results in partial oxidation of the Co layer (Figure S4).

\section{Conclusion}

The study of metal/organic hybrid heterostructures and especially of hybrid devices has proven to be overly complex because it is difficult to probe the continuity of organic molecular films embedded between ferromagnetic electrodes. In this work an original FNR methodology was proposed to investigate the morphology of organic layers embedded between Fe and Co ferromagnetic electrodes. When applied the FNR methodology to study the morphology of $\mathrm{Co} / \mathrm{ZnTPP} / \mathrm{Fe}$ and Fe/ZnTPP/Co stacks. The method showed that about 15 molecular layers are required to obtain heterostructures with continuous, pin hole free organic layers. In addition, 
the quantitative analyses of the FNR spectra showed that on Co, the ZnTPP layers show a layered morphology at small thickness, whereas on Fe, the molecules show island type of morphology. The FNR analyses led to a better understanding of the magnetization processes of the samples. This FNR methodology can be applied to any Co/spacer layer/Fe heterostructure and allows a deeper understanding of the morphology of embedded organic thin films.

\section{Experimental methods}

The investigated $\mathrm{Co} / \mathrm{ZnTPP} / \mathrm{Fe}$ heterostructures were prepared on $\mathrm{Si} / \mathrm{SiO}_{2}(500 \mathrm{~nm})$ substrates, on which a $25 \mathrm{~nm}$ copper $(\mathrm{Cu})$ seed layer was deposited by dc sputtering, under argon pressure of $4.5 \times 10^{-4}$ mbar in a standard sputtering chamber. After transfer of the sample (without breaking the vacuum) to an ultra-high vacuum (UHV) evaporation chamber, $6 \mathrm{~nm}$ thick layer of cobalt was deposited by thermal evaporation, with a flux of $0.16 \AA$. $\sec ^{-1}$ under deposition pressure of $2 \times 10^{-9}$ mbar. After Co deposition, the sample was transferred, without vacuum disruption, to the organic molecular beam epitaxy chamber (OMBE) for the preparation of the ZnTPP molecular layers. Analytically pure, in-lab synthesized ZnTPP molecules were sublimed on top of the ferromagnetic Co film under $2 \times 10^{-9}$ mbar deposition pressure and at a rate of 0.055 monolayer per seconds (ML. $\sec ^{-1}$ with $1 \mathrm{ML}=3.5 \AA$; monitored with quartz). Finally, the sample was transferred back to the sputtering chamber to prepare the iron $(\mathrm{Fe})$ counter electrode. A layer of $30 \mathrm{~nm}$ of iron $(\mathrm{Fe})$ was sputtered in dc sputtering mode, under argon deposition pressure of $4.5 \times 10^{-4}$ mbar and with a flux of $0.25 \AA$. $\mathrm{sec}^{-1}$. In the sputtering system the distance between the target and sample is of $0.25 \mathrm{~m}$, in order to avoid damaging of the organic layer by the plasma. In a similar way, Fe/ZnTPP/Co heterostructures were prepared first by sputtering the $30 \mathrm{~nm}$ of iron $\left(0.25 \AA . \mathrm{sec}^{-1}\right)$ on top of the copper seed layer prepared in the above-mentioned conditions. Next, the sample was transferred to OMBE for ZnTPP deposition. The molecular films were prepared using the same flux as above, to have similar growth condition as that of the $\mathrm{Co} / \mathrm{ZnTPP} / \mathrm{Fe}$ structures. Finally, $6 \mathrm{~nm}$ layer of cobalt was 
deposited $\left(0.16 \AA\right.$. $\left.\sec ^{-1}\right)$ as the upper electrode at room temperature and under deposition pressure of $2 \times 10^{-9}$ mbar. All layers in the heterostructures were capped with $100 \mathrm{~nm}$ of chromium (Cr).

All FNR measurements were performed in a homemade state of the art FNR set up. The integrated spin-echo intensity was recorded using a broadband pulsed spectrometer with phasesensitive detection and automated frequency scanning (pulse length: $3.2 \mu$ s, delay: $3 \mu$ s). The FNR spectra were taken for at least five different values of the excitation radiofrequency (RF) field power, covering a range of over more than one order of magnitude. Such a procedure allows the determination of the optimum excitation field power at each frequency and corrects the variation of the local electronic susceptibility, thus the of FNR enhancement factor as a function of frequency. ${ }^{[5]}$ After this, a further correction for the regular frequency dependence of the FNR signal was applied. The resulting FNR amplitudes represent the true distribution of the probed atoms (nuclei) versus the RF field frequency. The FNR measurements were performed at $2 \mathrm{~K}$ and the samples' surface area was of the order of $3 \mathrm{~cm}^{2}$. All the spectra have been averaged for 12 hours. As the thickness of the samples is much thinner than the RF penetration length, several micrometers for the considered frequency range, the full stack FNR signal contribution is retrieved. With these experimental conditions the set-up sensitivity is better than 0.1 atomic layer of Co and in integral form it is better than 0.01 atomic layers of Co (integrated over 100 significant data points). All the spectra have been normalized to the sample surface area.

The magnetic hysteresis loops were measured using the commercial MPMS3 SQUID (Quantum Design Inc) magnetometer operating in DC mode. All measurements were conducted at $2 \mathrm{~K}$ after field cooling procedure in an in-plane external static magnetic field of $+6 \mathrm{~T}$ (tesla). Sample surface area was of the order $12 \mathrm{~mm}^{2}$. 


\section{Acknowledgements:}

This work was funded by the French National Research Agency (ANR) through the Programme d'Investissement d'Avenir under contract ANR-11-LABX-0058_NIE within the Investissement d'Avenir program ANR-10-IDEX-0002-02. We acknowledge Christophe Kieber for technical assistance and the IdEx program of the University of Strasbourg for a scholarship for Garen Avedissian.

\section{Reference}

[1] M. Gruber, F. Ibrahim, S. Boukari, H. Isshiki, L. Joly, M. Peter, M. Studniarek, V. Da Costa, H. Jabbar, V. Davesne, U. Halisdemir, J. Chen, J. Arabski, E. Otero, F. Choueikani, K. Chen, P. Ohresser, W. Wulfhekel, F. Scheurer, W. Weber, M. Alouani, E. Beaurepaire, M. Bowen, Nat. Mater. 2015, 14, 981.

[2] S. Boukari, H. Jabbar, F. Schleicher, M. Gruber, G. Avedissian, J. Arabski, V. Da Costa, G. Schmerber, P. Rengasamy, B. Vileno, W. Weber, M. Bowen, E. Beaurepaire, Nano Lett. 2018, 18,4659 .

[3] W. Xu, G. J. Szulczewski, P. LeClair, I. Navarrete, R. Schad, G. Miao, H. Guo, A. Gupta, Appl. Phys. Lett. 2007, 90, 072506.

[4] J. M. Gottfried, Surf. Sci. Rep. 2015, 70, 259.

[5] P. Panissod, C. Meny, Appl. Magn. Reson. 2000, 19, 447.

[6] J. L. Bubendorff, E. Beaurepaire, C. Meny, J. P. Bucher, J. Appl. Phys. 1998, 83, 7043. 
[7] M. Vélez, C. Meny, S. M. Valvidares, J. Diaz, R. Morales, L. M. Alvarez-Prado, P. Panissod, J. M. Alameda, Eur. Phys. J. B. 2004, 41, 517.

[8] A. Azizi, A. Sahari, M. L. Felloussia, G. Schmerber, C. Meny, A. Dinia, Appl. Surf. Sci. 2004, 228, 320.

[9] M. Wójcik, J. P. Jay, P. Panissod, E. Jedryka, J. Dekoster, G. Langouche, Zeitschrift für Physik B. 1997, 103, 5.

[10] H. El Fanity, K. Rahmouni, M. Bouanani, A. Dinia, G. Schmerber, C. Meny, P. Panissod, A. Cziraki, F. Cherkaoui, A. Berrada, Thin Solid Films.1998, 318, 227.

[11] J. Dekoster, E. Jedryka, C. Meny, G. Langouche, J. Magn. Magn. Mater. 1993, 121, 69.

[12] K. Le Guen, M. -H. Hu, J. -M. Andre, S. K. Zhou, H. Ch. Li, J. T. Zhu, Z. S. Wang, C. Meny, A. Galtayries, P. Jonnard, Appl. Phys. Let. 2011, 98, 251909.

[13] E. Jedryka, M. Wójcik, S. Nadolski, D. Kubinski, M. Parsons, H. Holloway, J. Appl. Phys. 2002, 91, 7191.

[14] M. Wójcik, C. Christides, E. Jedryka, S. Nadolski, I. Panagiotopoulos, Phys. Rev. B. 2001, 63, 012102.

[15] C. Meny, P. Panissod, P. Humbert, J. P. Nozieres, V. S. Speriosu, B. A. Gurney, R. Zehringer, J. Magn. Magn. Mater. 1993, 121, 406.

[16] Y. F. Liu, J. Luo, Y. Shin, S. Moldovan, O. Ersen, A. Hebraud, G. Schlatter, C. PhamHuu, C. Meny, Nat. Commun. 2016, 7, 11532.

[17] A. S. Andreev, O. B. Lapina, J. B. d'Espinose de Lacaillerie, A. A. Khassin, J. Struct Chem, 2013, 54, S102.

[18] M. Malinowska, M. Wójcik, S. Nadolski, E. Jedryka, C. Meny, P. Panissod, M. Knobell, A. D. C. Viegas, J. E. Schmidt, J. Magn. Magn. Mater. 1999, 198-199, 599.

[19] R. Speight, A. Wong, P. Ellis, P. T. Bishop, T. I. Hyde, T. J. Bastow, M. E. Smith, Phys. Rev. B. 2009, 79, 054102.

[20] V. Scarani, H. De Riedmatten, J. P. Ansermet, Appl. Phys. Lett. 2000, 76, 903.

[21] C. Meny, E. Jedryka, P. Panissod, J. Phys. : Condens. Matter. 1993, 5, 1547.

[22] M. Malinowska, C. Meny, E. Jedryka, P. Panissod, J. Phys. : Condens. Matter. 1998, 10, 4919.

[23] H. v. Berlepsch, C. Böttcher, C., J. Phys. Chem. B. 2002, 106, 3146.

[24] T. Kobayashi, J-Aggregates, World Scientific, Singapore, 1996. 


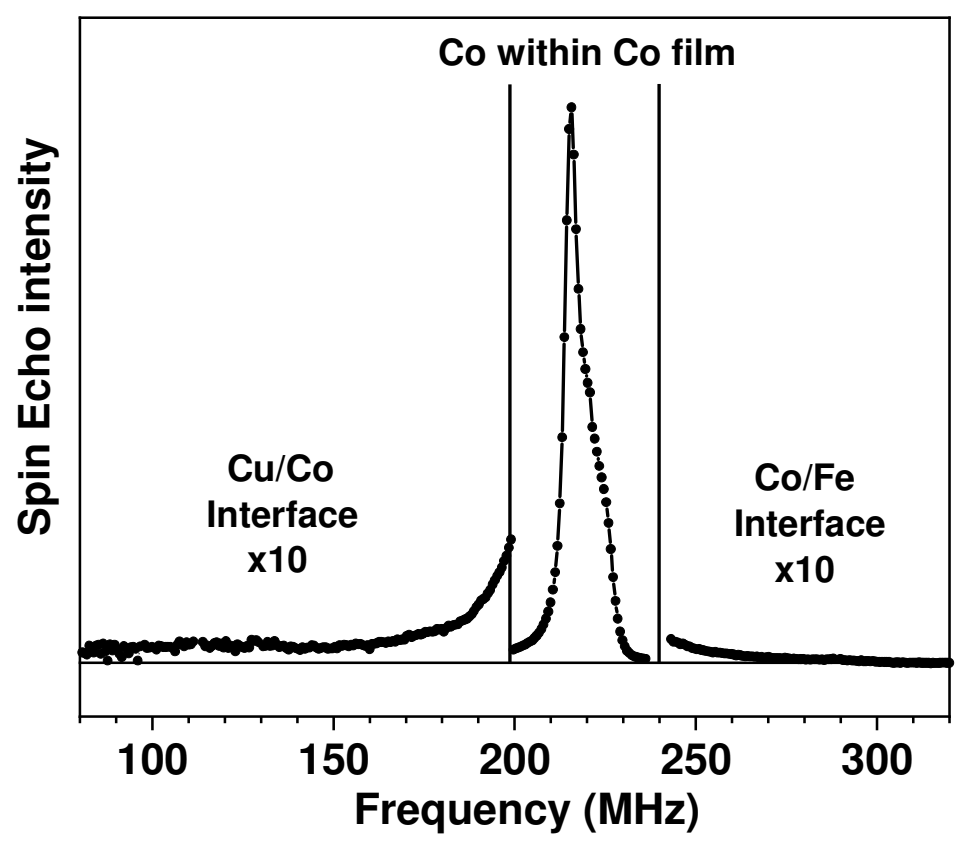

Figure 1: $\mathrm{FNR} \mathrm{Co}^{59}$ spectrum for $\mathrm{Cu} / \mathrm{Co} / \mathrm{Fe}$ sample. The spectrum shows three distinct regions separated by the solid lines: the low frequency intensity $(<200 \mathrm{MHz})$ is attributed to the $\mathrm{Cu} / \mathrm{Co}$ interfacial contributions, the central region $(200<$ frequency $(\mathrm{MHz})<240)$ corresponds to metallic cobalt atoms inside the cobalt film, and the high frequency intensity $(>240 \mathrm{MHz})$ originates from the $\mathrm{Co} / \mathrm{Fe}$ interface. Spin echo intensities for low and high frequency ranges are magnified 10 times (x10) for clarity.

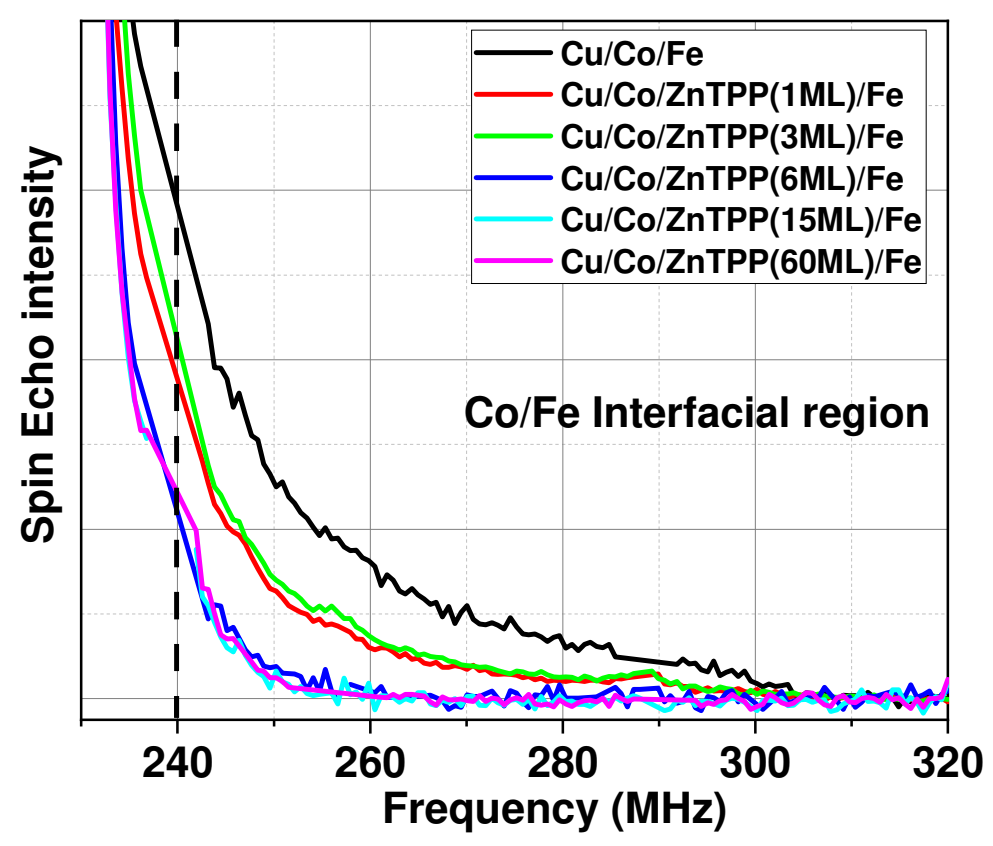


Figure 2: Evolution of high frequency (>240 MHz) spectral range of $\mathrm{Co}^{59} \mathrm{FNR}$ spectra with increasing thickness of the ZnTPP molecular layers. The high frequency contribution decreases with the increase in the ZnTPP thickness (1 to $60 \mathrm{ML}$ ) and vanish from $15 \mathrm{ML}$ and above.

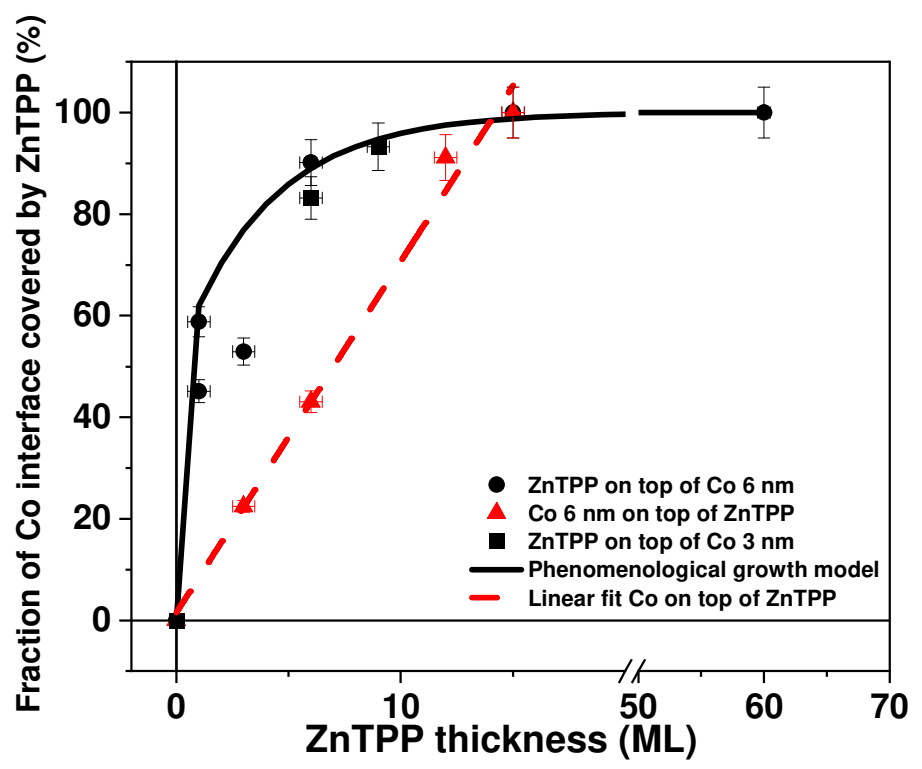

Figure 3: The evolution of ZnTPP molecular coverage on top of Co and Fe metallic surfaces, with the increase in ZnTPP thickness. Black square and circle symbols represent the experimental data of ZnTPP coverage ratio when deposited on top of Co. Black line is the fit using phenomenological growth model. Red triangles represent ZnTPP coverage ratio when deposited on top of Fe. Red dashed line is the linear fitting of ZnTPP coverage rate on Fe. 


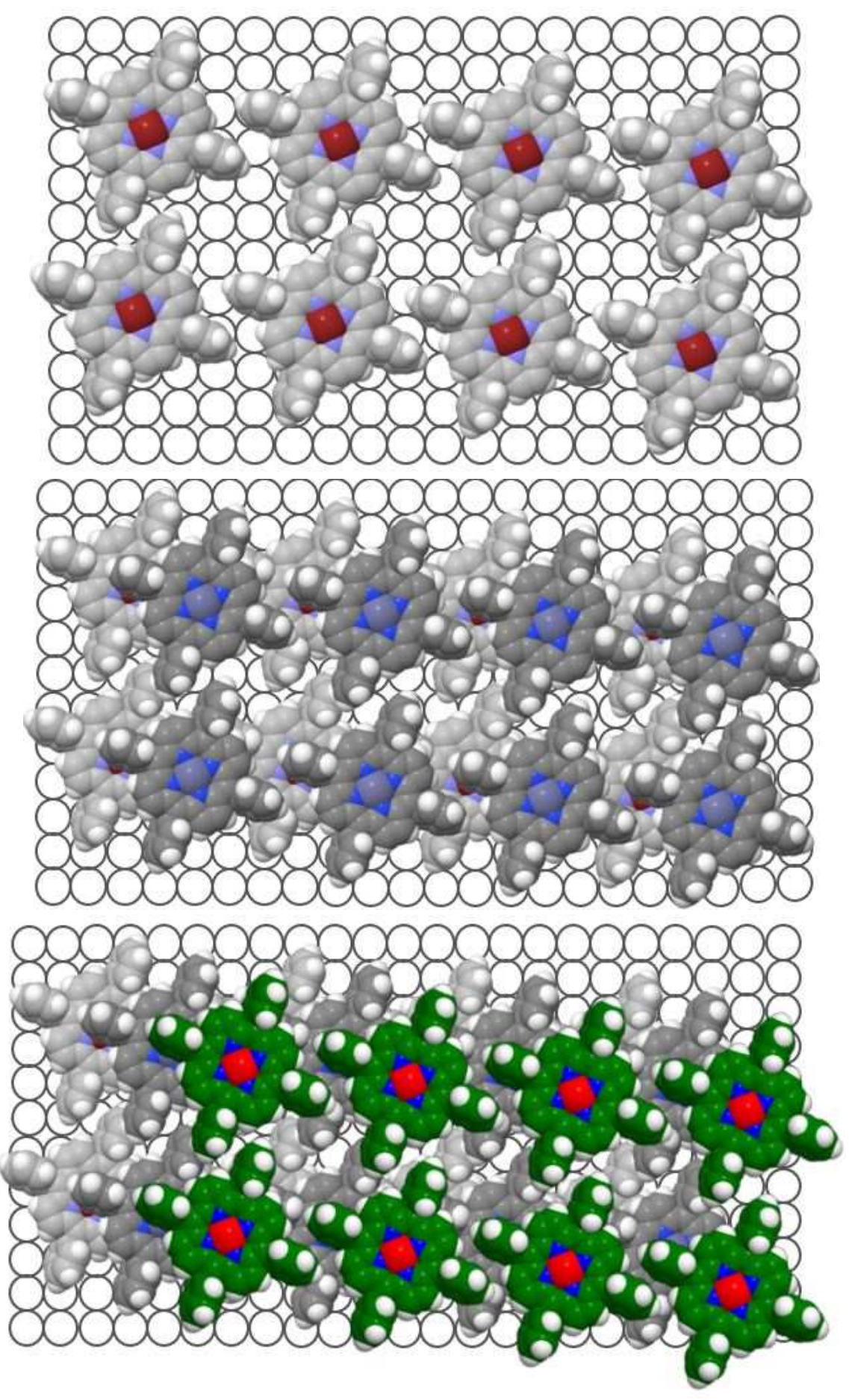

Figure 4: Proposed schemes for the ZnTPP morphology on a metallic surface. Top panel: 1ML of ZnTPP (light grey molecules) organized on top of a square metallic lattice. The ratio of metallic atoms (white circles) covered by the molecules is estimated to be of the order of $62 \%$. Middle panel: stacking of the $2^{\text {nd }}$ ML (dark grey molecules) leads to $85 \%$ coverage ratio. Bottom panel: stacking of the $3^{\text {rd }}$ ML (green molecules) results in almost full coverage of the metallic surface. In all these schemes, the molecules are arranged as such they respect the maximum $\pi-\pi$ stacking and the offset for a typical J-aggregate. 


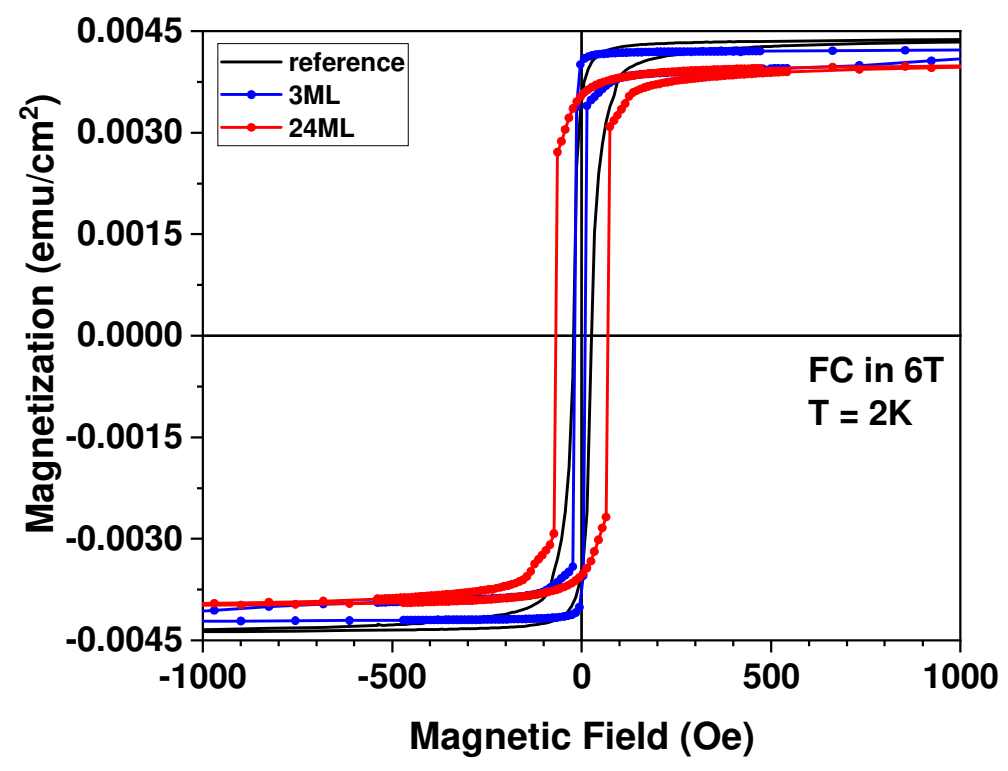

Figure 5: In-plane magnetization loops. Black line: $\mathrm{Cu} / \mathrm{Co} / \mathrm{Fe}$ reference sample. Blue line: Co/ZnTPP (3 ML)/Fe heterostructure. Red line: Co/ZnTPP (24 ML)/Fe sample. All measurements are made at $2 \mathrm{~K}$ after $\mathrm{FC}$ in in-plane $+6 \mathrm{~T}$ external magnetic field. 
Probing the growth of organic molecular films embedded between cobalt and iron electrodes: Ferromagnetic nuclear resonance approach

Garen Avedissian*, Jacek Arabski, Jennifer A. Wytko, Jean Weiss and Christian Meny*

Figure S1 shows the full radiofrequency spectral range of $\mathrm{Co}^{59} \mathrm{FNR}$ spectra of $\mathrm{Cu}(25 \mathrm{~nm}) / \mathrm{Co}$ $(6 \mathrm{~nm}) / \mathrm{ZnTPP}$ ( $t$ in $M L) / \mathrm{Fe}(30 \mathrm{~nm})$ heterostructures with 1, 3, 6, 15 and 60 monolayers of ZnTPP compared to the reference sample. Here, the overall behaviour of the heterostructures is observed showing the two interfacial contribution in the FNR spectra. Low frequency range (<200 MHz) corresponds to $\mathrm{Cu} / \mathrm{Co}$ interfacial contribution while the high frequency range (> $240 \mathrm{MHz}$ ) corresponds to $\mathrm{Co} / \mathrm{Fe}$ interfacial contribution. As observed and explained, the CoFe contribution decreases when the ZnTPP thickness increases and eventually vanishes

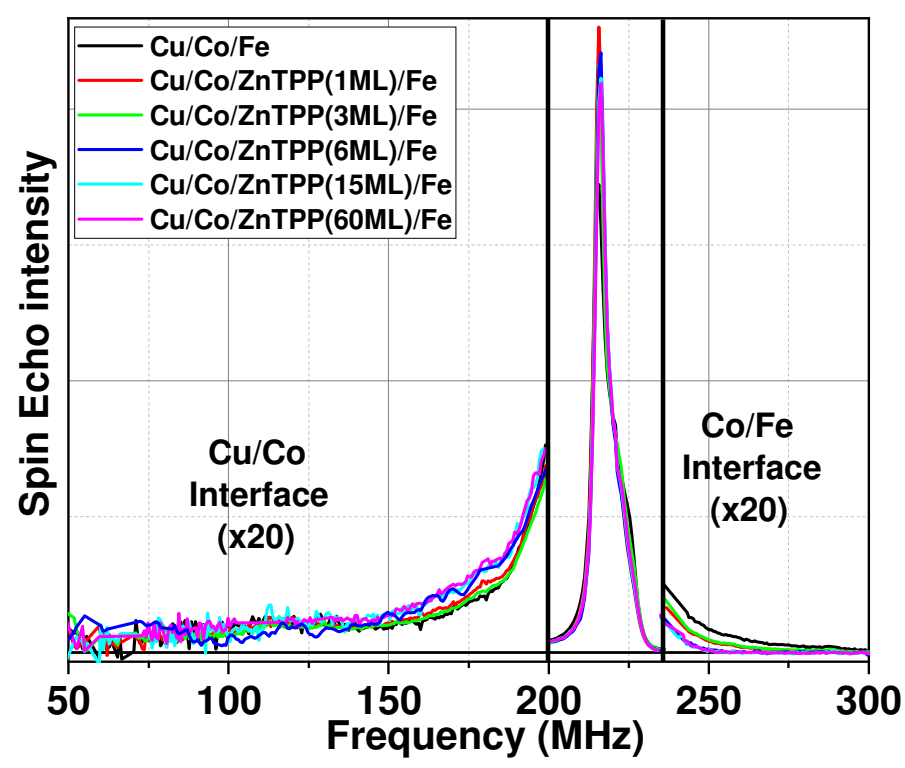

Figure S1. Full radiofrequency spectral range representation for Co (6nm)/ZnTPP (t in ML)/ $\mathrm{Fe}(30 \mathrm{~nm})$ heterostructures. Spin echo intensities for low and high frequency ranges are magnified 20 times (x20) for clarity. 
Figure S2 represents the $\mathrm{Co}^{59} \mathrm{FNR}$ spectra of $\mathrm{Cu}(25 \mathrm{~nm}) / \mathrm{Co}(3 \mathrm{~nm}) / \mathrm{ZnTPP}$ ( $t$ in $\left.M L\right) /$ $\mathrm{Fe}(30 \mathrm{~nm})$ heterostructures with 6 and 9 monolayers of ZnTPP, compared to the reference sample. Only high frequency spectral range is presented. As observed and explained in the main text, the intensity of the high frequency range (> $240 \mathrm{MHz}$ ) decreases when 6 and 9 molecular layers of ZnTPP are introduced between the cobalt and iron films. This observation is consistent with the explained FNR methodology.

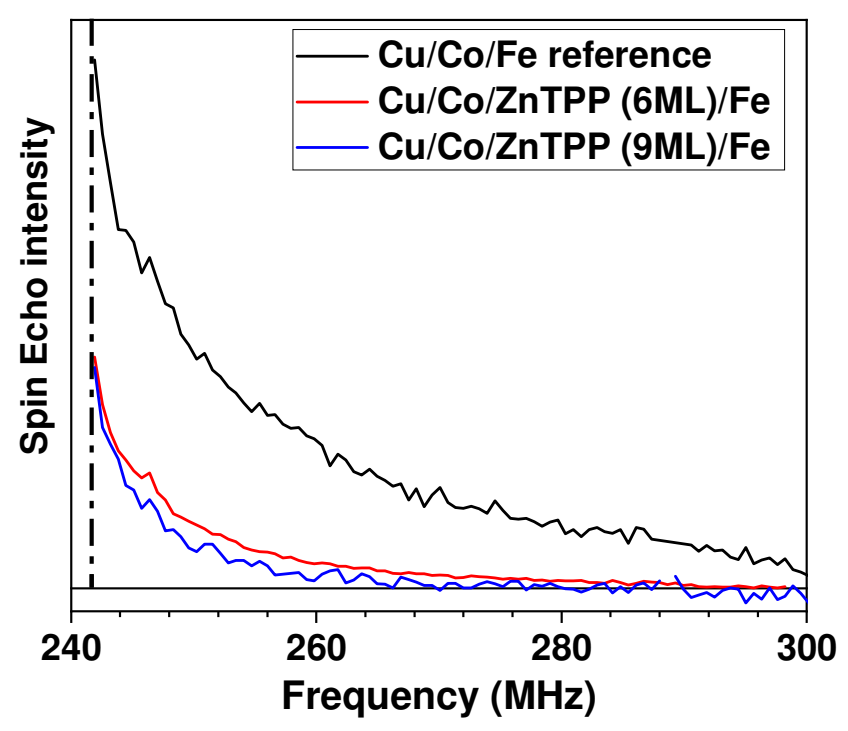

Figure S2. High frequency representation for Co (3nm)/ZnTPP (t in ML)/ Fe (30nm) heterostructures.

In Figure S3, when the order of the electrodes in the samples is reversed ZnTPP molecules are grown on top $\mathrm{Fe}(30 \mathrm{~nm})$, while the counter electrode deposited on top of the molecules is Co (6nm). Again, intensity in the frequency range above $240 \mathrm{MHz}$ decreases when ZnTPP thickness increase. The high frequency contributions vanish for 15 ML of ZnTPP (dark green spectrum). 


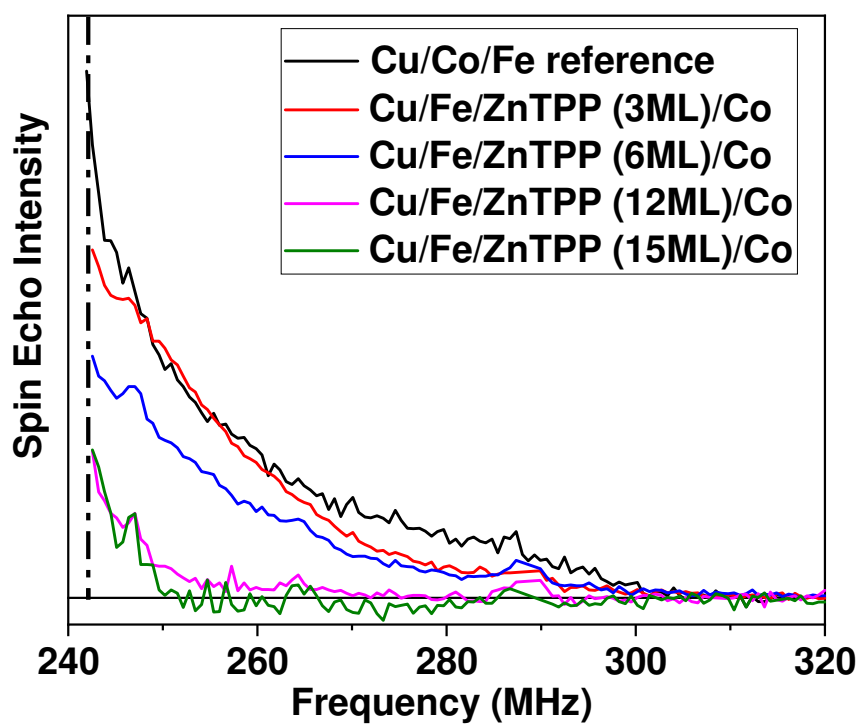

Figure S3. High frequency representation for Fe (30nm) /ZnTPP ( $\mathrm{t}$ in ML)/Co (6nm) heterostructures.

Figure S4 shows the magnetization loop of Co (6nm)/ZnTPP (60ML)/ Fe (30nm)/Cr (100nm) sample, measured right after removal from the UHV chamber (fresh sample, black hysteresis) and measured again for a second time after 1 year (red loop). As observed, the freshly measured sample shows similar magnetic behavior as the Co/ZnTPP (24ML)/Fe sample. At smaller fields, the magnetization of the iron is reversed while the reversal at higher fields is attributed to the magnetically stiffer cobalt. However, when the sample is measured again after 1 year, it showed different behavior than its initial state. Enhancement of coercive field in each reversal was observed. More strikingly, the stiffer region, corresponding to the cobalt film, shows asymmetrical reversal, thus the onset of exchange bias. This observation suggests that, the presence of exchange bias in these hybrid structures originates from the partial oxidation of cobalt film, when the samples are exposed to air. 


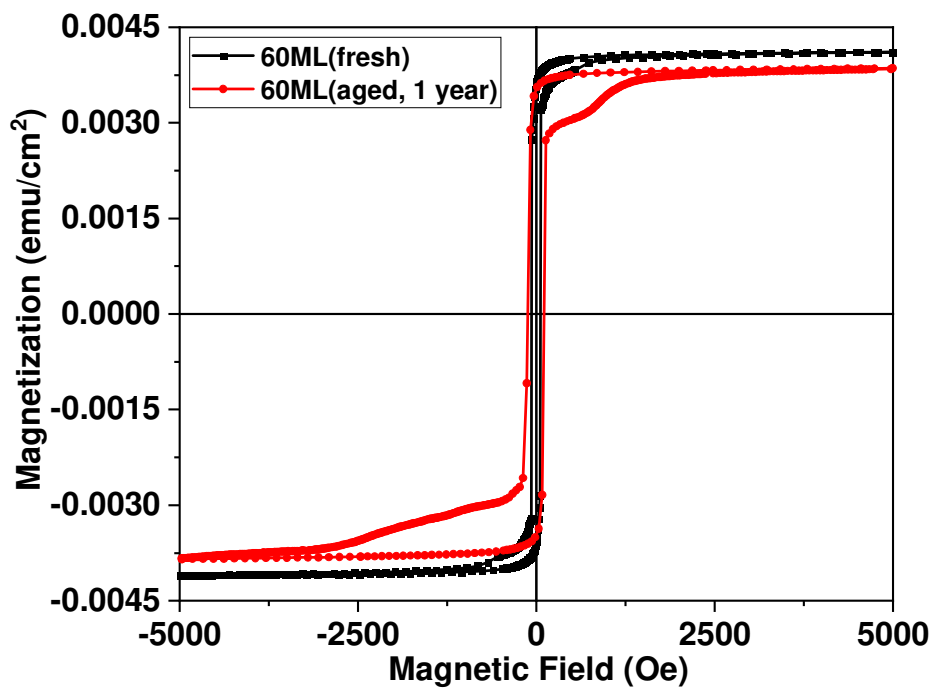

Figure S4. Magnetic hysteresis loop of Co (6nm)/ ZnTPP (60ML)/ Fe (30nm) sample. Black loop: directly after growth, red loop: measured again after 1 year. Measurements are done at $2 \mathrm{~K}$ after $\mathrm{FC}$ in $+6 \mathrm{~T}$ in-plane magnetic field. 